\title{
Knowledge and behavioural interventions to reduce human health risk from private groundwater systems: A global review and pooled analysis based on development status
}

\author{
S. Mooney \\ Technological University Dublin \\ C. P. McDowell \\ University College Dublin, Ireland \\ J. O'Dwyer \\ University College Cork, Ireland
}

See next page for additional authors

Follow this and additional works at: https://arrow.tudublin.ie/ehsiart

Part of the Environmental Health and Protection Commons, Environmental Public Health Commons, Natural Resources and Conservation Commons, Natural Resources Management and Policy Commons, Other Environmental Sciences Commons, and the Water Resource Management Commons

\section{Recommended Citation}

Mooney S, McDowell CP, O'Dwyer J, Hynds PD. Knowledge and behavioural interventions to reduce human health risk from private groundwater systems: A global review and pooled analysis based on development status. Sci Total Environ. 2020 May 10;716:135338. doi: 10.1016/j.scitotenv.2019.135338. Epub 2019 Nov 23. PMID: 31839297.

This Article is brought to you for free and open access by the ESHI Publications at ARROW@TU Dublin. It has been accepted for inclusion in Articles by an authorized administrator of ARROW@TU Dublin. For more information, please contact arrow.admin@tudublin.ie, aisling.coyne@tudublin.ie,gerard.connolly@tudublin.ie.

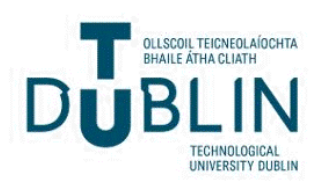


Authors

S. Mooney, C. P. McDowell, J. O'Dwyer, and P. D. Hynds

This article is available at ARROW@TU Dublin: https://arrow.tudublin.ie/ehsiart/20 


\title{
Knowledge and behavioural interventions to reduce human health risk from private groundwater systems: A global review and pooled analysis based on development status
}

\author{
S. Mooney ${ }^{\mathrm{a}}$, C.P. McDowell ${ }^{\mathrm{b}}$, J. O’Dwyer ${ }^{\mathrm{c}, \mathrm{d}, \mathrm{e}}$, P.D. Hynds ${ }^{\mathrm{a}, \mathrm{e}, *}$ \\ ${ }^{a}$ Environmental Sustainability \& Health Institute, Technological University Dublin, Dublin, Ireland \\ ${ }^{\mathrm{b}}$ School of Architecture, Planning and Environmental Policy, University College Dublin, Dublin, Ireland \\ ${ }^{\mathrm{C}}$ School of Biological, Earth and Environmental Sciences, University College Cork, Cork, Ireland \\ ${ }^{\mathrm{d}}$ Environmental Research Institute, University of Cork, Cork, Ireland \\ ${ }^{\mathrm{e}}$ Irish Centre for Research in Applied Geosciences (iCRAG), University College Dublin, Dublin, Ireland
}

\section{H I G H L I G H T S}

- PICO review identified 40 distinct private groundwater interventions.

- Just one identified intervention associated with microbial contamination of private groundwater.

- Mean behavioural and knowledge attainment across interventions was $53 \%$ and $48 \%$, respectively.

- Interventions in economically developed regions exhibited higher behavioural outcomes.

- Practical interventions allied with both large- and local-scale awareness-raising campaigns represent an optimum approach.

\section{A R T I C L E I N F O}

\section{Article history:}

Received 29 August 2019

Received in revised form 31 October 2019

Accepted 31 October 2019

Available online 23 November 2019

\section{G R A P H I C A L A B S T R A C T}

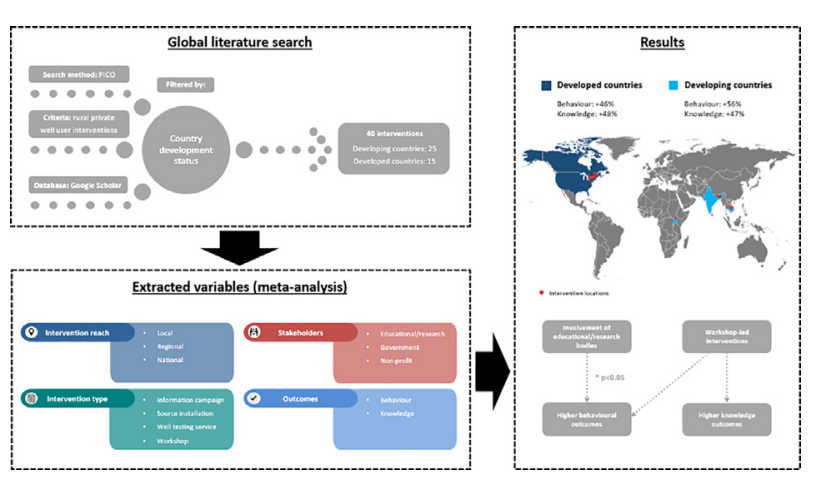

\section{A B S T R A C T}

Groundwater contamination constitutes a significant health risk for private well users residing in rural areas. As the responsibility to safeguard rural private domestic groundwater typically rests with nonexpert homeowners, interventions promoting risk mitigation and awareness represent the most viable means of preventing supply contamination. However, no global review or pooled analyses of these interventions has been undertaken to date. The current study sought to identify and quantify the performance of private well interventions from 1990 to 2018 via a global systematised review and pooled analysis. The PICO (Population-Intervention-Comparison-Outcome) approach was employed for literature identification. Relevant studies were statistically analysed across two quantitative outcome (performance) types, namely knowledge and behaviour, controlling for intervention characteristics and country development status. Mean behavioural and knowledge attainment across interventions was $53 \%$ and $48 \%$, respectively, with interventions in economically developed regions exhibiting higher behavioural outcomes (56\% vs. $45 \%$ ) than those in developing regions. Geographically, interventions were located in southern or southeast Asia $(n=23)$, North America $(n=15)$, Central America $(n=1)$ and Africa $(n=1)$, with none identified in Australia/Oceania, Europe, or South America. Behavioural outcomes were significantly associated with presence of educational/research coordinator $(p=0.023)$, with these interventions attaining higher levels of efficacy $(+74 \%)$ than those implemented by other coordinator types. Findings indicate that instructor-

\footnotetext{
* Corresponding author.

E-mail address: hyndsp@tcd.ie (P.D. Hynds).
} 
led, practical interventions allied with both large- and local-scale awareness-raising campaigns represent an optimum approach for future private well risk interventions. Subsequent adoption of such interventions may lead to increased levels of private well maintenance and provide a point of reference for myriad water and health communication contexts.

(c) 2019 Elsevier B.V. All rights reserved.

\section{Introduction}

Groundwater is the most extracted raw material on earth and represents the largest global reservoir of accessible freshwater for human consumption, currently serving an estimated 2.2 billion people (Gleeson et al., 2016; Murphy et al., 2017). With a significant portion of historical groundwater abstraction occurring in the last 50 years due to rising populations and dwindling surface water resources, established reserves have become overexploited (Gorelick \& Zheng, 2015). As a result, groundwater quality has become severely compromised in many regions, with improved supply management now regarded as a key environmental health challenge for the $21^{\text {st }}$ century (Gorelick \& Zheng, 2015; Jakeman et al., 2016). Much of the recent research pertaining to global groundwater management has focused on predicting and modelling aquifer withdrawals and developing options for recovery and restoration (Niu et al., 2014). Of more immediate concern is the widespread susceptibility of potable sources to contamination posing human health risks, particularly in light of shifting land-use patterns and climate change (Green et al., 2011; O'Dwyer et al., 2016; Andrade et al. 2018).

Unprecedented rates of groundwater abstraction in areas characterised by high geochemical concentrations, expanding agroindustrial activities and climate change-induced changes in precipitation patterns have resulted in the wide proliferation of chemical and microbial contaminants in groundwater supplies (Schwarzenbach et al., 2010; Gorelick \& Zheng, 2015). An increasing number of well users worldwide have consequently suffered severe health impacts due to chronic exposure to naturally occurring chemicals such as arsenic and nitrate and contraction of waterborne diseases (Jakeman et al., 2016). Global estimates suggest that over 150 million people currently ingest arsenic from groundwater drinking supplies exceeding permissible limits set by the WHO (Ravenscroft et al., 2009). Moreover, a recent global review of groundwater-related enteric disease outbreaks identified 649 events within the published literature during the period 19482015 and a steadily increasing incidence of groundwater-related acute gastrointestinal infections (AGI) (Murphy et al., 2017). Rural populations relying on private, unregulated wells are particularly susceptible to illness due to the wide range of proximate contaminant sources (e.g. domestic wastewater treatment systems (DWWTSs), agriculture) and geographical/infrastructural obstacles (e.g. isolation from major population centres and services) hindering "top-down" groundwater management and policy implementation (Pearson et al., 2011; Valle Junior et al., 2014). Accordingly, private groundwater interventions (i.e. initiatives to promote source safety and protection) are being increasingly implemented in rural areas to compensate for the widespread absence of regulatory tools (Morris et al., 2016).

While many groundwater management challenges are frequently associated with socioeconomically "developing" regions, concomitant concerns are also reported in the more affluent "developed" nations (Shankar et al., 2014; WHO/UNICEF, 2015). Additionally, some of the most prevalent groundwater contaminants (e.g. naturally occurring arsenic) encountered in developing countries (e.g. Bangladesh) also occur in high concentrations in developed countries (e.g. United States) (Shankar et al., 2014; Henry \& Suk, 2018). Conversely, rural regions in developed countries are associated with their own suite of potential hazards to private groundwater; for example, DWWTS-originated contamination stemming from rapid exurban housing development and emerging organic contaminants (EOCs), e.g. industrial compounds, and intensive agriculture (Lapworth et al., 2012). In order to effectively minimise groundwater contamination and consequent health impacts on a global scale, it is important to acknowledge both regional commonalities and variations.

Due to the absence of private groundwater regulation in many territories, the onus to protect private rural groundwater largely lies with non-expert owners and users. Interventions entailing public engagement (e.g. information campaigns, well testing services) to promote protective behaviours and risk awareness may therefore constitute the most feasible approach to ensuring improved rural groundwater quality - irrespective of region of implementation (Kreutzwiser et al., 2011; Mitchell et al., 2012). The necessity for these initiatives has been highlighted in several previous studies reporting pronounced shortcomings in risk awareness and protective actions, with many recommending renewed or novel outreach strategies (Charrois, 2010; Malecki et al., 2017). However, in spite of the growing acknowledgement that enhanced engagement with private well users is necessary, benchmarks for effective private groundwater interventions (leading to "bottom-up" mitigation) remain elusive and existing frameworks inadequate (Mitchell et al., 2012; Hynds et al., 2018). Quantification of current success rates and outcome predictors associated with rural groundwater interventions may represent a benchmark for future hydrogeological investigations and management strategies addressing the intersect between groundwater and end-users. While previous studies have identified contextual factors surrounding well user outreach (e.g. Morris et al., 2016), to date, no broad empiric analysis of rural groundwater interventions and associated behavioural and knowledge outcomes exists in the literature. The current study responds to this deficit via a global systematised review and pooled-analysis to establish the performance of private well user interventions designed to engender increased source protection and risk awareness.

\section{Methods}

A systematised scoping review protocol was adapted and developed from previous studies (Elamin et al., 2009; Hynds et al., 2014). The review protocol was accepted and registered on PROSPERO (Registration Number CRD42017078019), and comprised four distinct process steps, namely: Literature Identification (Section 2.1), Study Selection (Section 2.2), Data Extraction (Section 2.3) and Pooled Analysis (Section 2.4).

\subsection{Literature identification}

The first stage of an evidence-based review process comprises development of an answerable question to facilitate identification of relevant literature (Moher et al., 2009). The present review was guided by the following research question:

What interventions have been employed to improve awareness and protective behaviour(s) among rural private groundwater users and what levels of success have been achieved? 
Due to the significant public health dimension of private groundwater interventions, the research question and literature identification process employed the PICO (Population, Intervention, Comparison, Outcomes) approach (Schardt et al., 2007; Higgins \& Green, 2011). The PICO method and its application within the current study, including explicit search terms and associated PICO components, are presented in Figure 1 and Table 1. In light of the multidisciplinary and global span of the review and consequent requirement for wide journal coverage, potentially relevant literature was searched using the Google Scholar search engine. While the authors acknowledge the weaknesses of Google Scholar as a systematic search tool (e.g. limited search algorithms and filters), assuring a blanket aggregation of studies was considered optimal for maximum article retrieval and deemed to negate these limitations (Haddaway et al., 2015). Databases characterised by a comparatively specialised, restricted focus (e.g. Scopus, PubMed) were hence not consulted (Falagas et al., 2008). A review period of 29 years (1990-2018) was used to ensure comprehensive capture of temporally relevant data. Search combinations incorporated a minimum of four individual search terms (one per PICO component) (Table 1).

Explicit eligibility (inclusion/exclusion) criteria were used for article screening (Table 2). Only peer-reviewed academic journal articles written in English evaluating measurable interventions intended for private groundwater supply users during or after 1990 were considered. Grey literature (e.g. technical reports, working papers, conference papers/abstracts, etc.) was excluded to avoid publication bias, skewed intervention coverage, and/or inadequate study design. Investigations located in non-rural (i.e. urban or peri-urban) areas and/or failing to present demarcated quantitative data specific to private well users were not considered appropriate for inclusion. Likewise, interventions not specifically directed at private groundwater sources and/or users were excluded.

\subsection{Study selection (screening)}

The literature search (SM, CM) returned 15,946 potentially relevant studies, of which 4,080 were retained for screening based on eligibility (Table 2). The first stage of literature screening was based on an independent assessment of article titles and abstracts (Figure 2), with 279 abstracts deemed to fulfil inclusion criteria. "Legacy" searches were subsequently undertaken to ensure identification of studies undetected during electronic searches. Follow-
Table 1

Literature review search terms and correspondent PICO classification and description.

\begin{tabular}{|c|c|c|}
\hline $\begin{array}{l}\text { Primary } \\
\text { Term }\end{array}$ & Term Description & Search Terms \\
\hline Population & $\begin{array}{l}\text { Rural private groundwater } \\
\text { supply (borehole, spring or } \\
\text { well) users }\end{array}$ & $\begin{array}{l}\text { Borehole users; groundwater } \\
\text { supply; private groundwater; } \\
\text { private well owners; spring } \\
\text { water; tube wells }\end{array}$ \\
\hline Intervention & $\begin{array}{l}\text { National and subnational } \\
\text { outreach strategies } \\
\text { targeting private well users } \\
\text { (information campaigns, } \\
\text { workshops etc.) }\end{array}$ & $\begin{array}{l}\text { Education; engagement; } \\
\text { information campaign; } \\
\text { intervention; outreach; risk } \\
\text { communication }\end{array}$ \\
\hline Comparison & $\begin{array}{l}\text { Differences between } \\
\text { interventions in type, } \\
\text { region, reach etc. }\end{array}$ & $\begin{array}{l}\text { Contamination; inspection; } \\
\text { registration; sampling; } \\
\text { testing; treatment }\end{array}$ \\
\hline Outcomes & $\begin{array}{l}\text { Impact on well user } \\
\text { behaviour and knowledge }\end{array}$ & $\begin{array}{l}\text { Awareness; behaviour; } \\
\text { knowledge; risk prevention; } \\
\text { risk reduction }\end{array}$ \\
\hline
\end{tabular}

Table 2

Eligibility (inclusion/exclusion) criteria employed for literature screening.

\begin{tabular}{lll}
\hline & Inclusion criteria & Exclusion criteria \\
\hline Literature & $\begin{array}{l}\text { Peer-reviewed academic } \\
\text { journal articles }\end{array}$ & Grey literature \\
Population & $\begin{array}{l}\text { English } \\
\text { Private groundwater supply } \\
\text { users residing in rural areas }\end{array}$ & $\begin{array}{l}\text { Non-English } \\
\text { Surface water and public } \\
\text { groundwater users }+ \\
\text { households residing in urban } \\
\text { or peri-urban areas } \\
\text { Evaluation }\end{array}$ \\
& $\begin{array}{l}\text { Quantitative investigations of } \\
\text { interventions intended to } \\
\text { promote groundwater supply } \\
\text { or non-quantitative data } \\
\text { relating to intervention } \\
\text { outcomes } \\
\text { Period }\end{array}$ & $\begin{array}{l}\text { 1990-present } \\
\text { Pre-1990 (intervention and } \\
\text { article) }\end{array}$ \\
\hline
\end{tabular}

ing omission of grey literature (-90 studies) and addition of relevant journal article abstracts/titles from study reference lists (+81 studies), 270 articles remained for the second phase of literature screening (full article analysis).

Full articles were analysed using established eligibility criteria, with studies independently assessed by three authors (SM, CM, PH) and, where divergence arose, iteratively deliberated until consensus was reached. The final stage of screening was predicated on suitability of interventions and evaluations, resulting in the inclusion of 31 articles containing 40 distinct interventions.

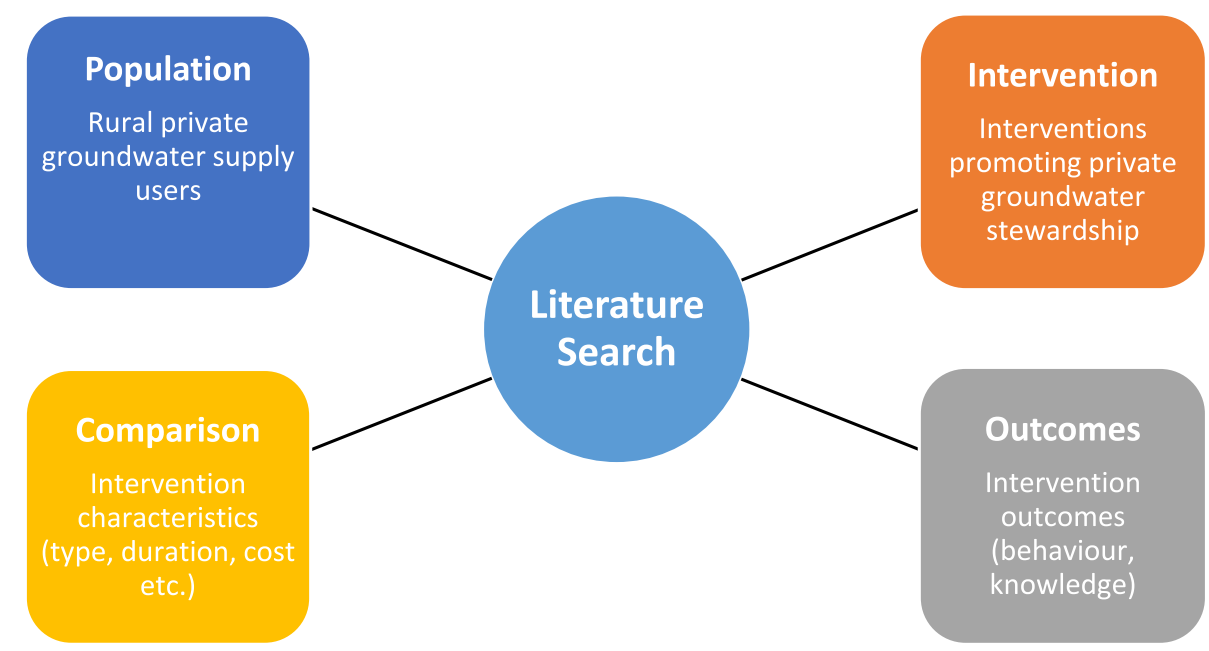

Figure 1. The PICO approach as applied for literature identification and data extraction in the current study. 


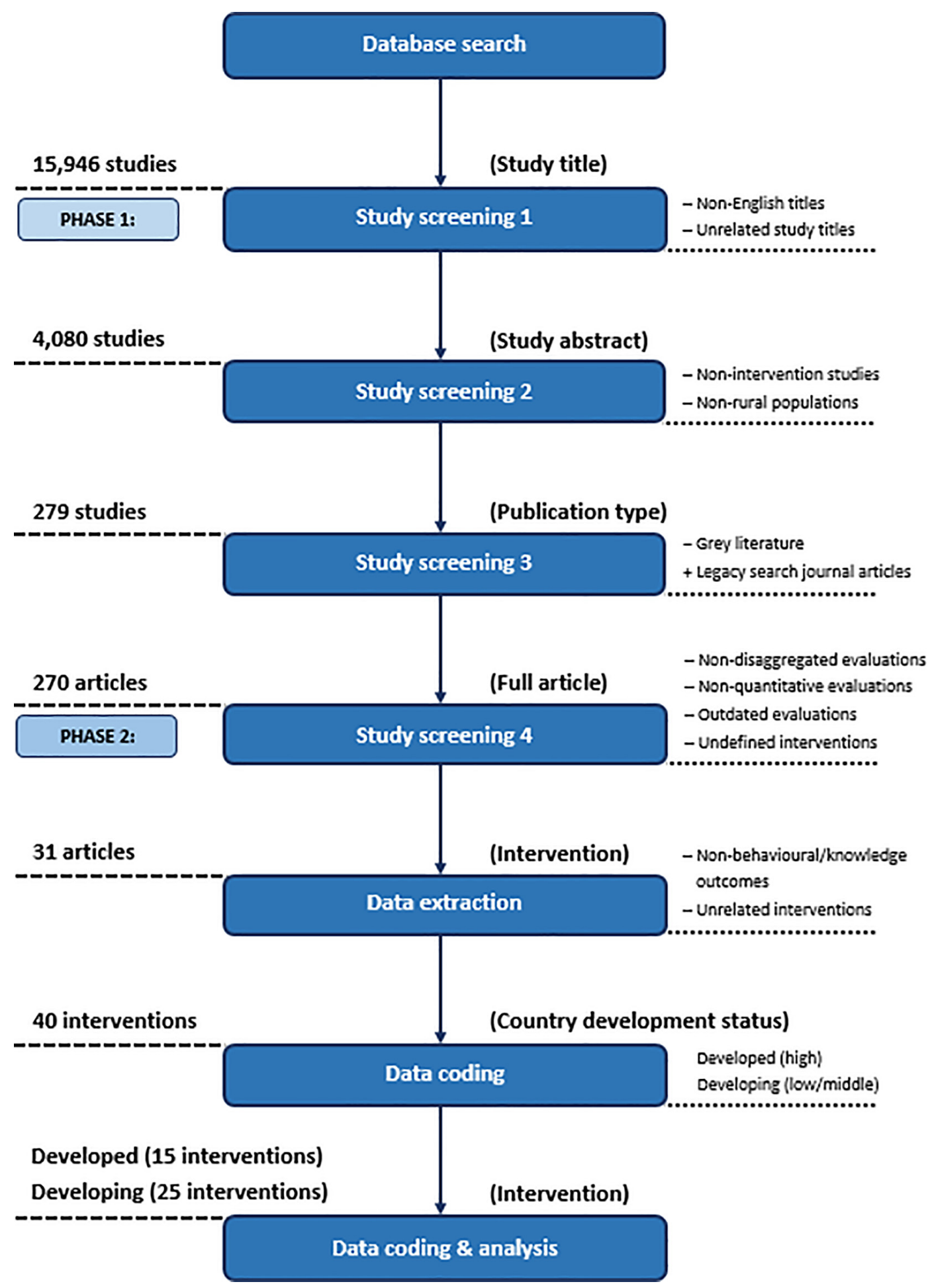

Figure 2. Schematic of study selection protocol.

\subsection{Data extraction}

Overall, 74 variables were extracted under nine primary categories (Appendix). Interventions were coded with respect to country development status following the emergence of a natural dichotomy between high and low/medium development nations. The separation was deemed to increase accessibility for prospective private groundwater intervention coordinators, as interventions in countries of contrasting socio-economic interest, notwithstanding potential similarities/transferability, are likely to differ in orientation and effectiveness (Oxman et al., 2009; Waddington et al., 2012).

Intervention reach (i.e. geographic extent of influence) was categorised with reference to the WHO's ExpandNet Framework for intervention scaling and divided into three classifications: large (national/state/regional), medium (sub-regional/county) and small (community/local) (WHO, 2009). Intervention mechanisms (i.e. constituent activities of overarching interventions) were categorised based on public engagement typologies developed by Rowe \& Frewer (2005), with two mechanism types (electronic 
and print) based upon uni-directional information flow from intervention coordinator to audience (recipient population) and one mechanism type (interpersonal) based upon reciprocal (bidirectional) information flow. A fourth mechanism type (convenience) was designated for intervention activities entailing direct support (e.g. provision of contaminant treatment system) and/or practical action (e.g. well installation) on behalf of well users. Classification of intervention stakeholders, i.e. coordinators (bodies responsible for implementing interventions) broadly emulated the stakeholder classes set out by Bunn et al. (2002), as follows: community body, educational/research body, government body, nonprofit body and other body. Extracted outcomes comprised measured behaviours and knowledge levels explicitly related to well safety, maintenance and health risks and were selected based on their assigned importance to intervention objectives by study authors. Where primary behavioural and knowledge outcomes were not specified, multiple measures were combined and averaged for each respective outcome type.

Dummy variables were developed for seven data categories; in instances where relevant information (e.g. regulatory status of private groundwater supplies in intervention area) was not provided in articles but available elsewhere, relevant sources (e.g. UNDP Human Development Index) were consulted (UNDP, 2016). For numerical (continuous) variables (e.g. intervention duration), intervals and units of measurement were discretised into appropriate ranges. Upon completion of data extraction and division of interventions by country development status, 69 variables were retained for coding and analyses, with five variables (hydrogeological setting, intervention cost, presence of disease/illness, number of wells, well depth) excluded due to incomplete records (available in $<50 \%$ of studies).

\subsection{Pooled Analysis}

Data were numerically coded and imported to IBM SPSS Statistics 26 (Armonk, NY: IBM Corp) for statistical analyses. Descriptive statistical functions (i.e. box and whisker plots displaying the median, interquartile ranges and present extreme/outlier values and stem-and-leaf plots) were employed to detect outliers across results per outcome type (behaviour and knowledge). Upon removal of outliers, outcomes were analysed for normality through visual inspection of Q-Q plots and the Shapiro-Wilk test. As overall outcomes followed a normal distribution, parametric statistical testing was employed.

The Pearson chi-square test of independence was used to test for associations between dichotomous (e.g. developed/developing) and nominal (e.g. intervention type) categorical variables. Oneway analysis of variance (ANOVA) tests were used to explore relationships between intervention characteristics (categorical variables) and outcomes (continuous variables). One-way ANCOVAs examined adjusted associations (study design and country development status) between intervention characteristics and knowledge and behaviour outcomes. Study design denotes presence or absence of baseline data in intervention evaluations. The level of significance was set at $\mathrm{p}<0.05$ by convention.

\section{Results}

\subsection{Intervention characteristics}

The review process yielded 40 distinct interventions (Figure 2), of which $58 \%(n=23)$ were undertaken in southern or southeastern Asia, with remaining interventions from the United States (28\%, $\mathrm{n}=11)$, Canada $(10 \%, \mathrm{n}=4)$, Guatemala $(3 \%, \mathrm{n}=1)$ and Uganda $(3 \%, n=1)$ (Table 3$)$. Notably, no interventions from Eur- ope, South America or the Australian sub-continent were identified (Figure 3). Chemical and microbial contaminants were reported in $83 \%(n=33)$ and $3 \%(n=1)$ of interventions, respectively, with remaining studies failing to specify contaminant type. Drilled boreholes comprised the majority $(80 \%, n=32)$ of private groundwater supplies for which interventions were reported. Well depths and local/regional (hydro)geological information was reported for just $18 \%(n=7)$ and $13 \%(n=5)$ of interventions, respectively. Six interventions were undertaken in areas currently subject to private groundwater regulation (United States), including mandatory well water testing transactions $(10 \%, \mathrm{n}=4)$ and well construction standards $(5 \%, n=2)$.

Seven intervention types (i.e. distinct outreach strategies) were identified, with information campaigns $(65 \%, \mathrm{n}=26$ ) and well testing services $(65 \%, \mathrm{n}=26)$ the most frequently implemented (Table 4). With respect to intervention reach, interventions were most often oriented at the subregional/county (medium) scale ( $43 \%, n=17$ ). Of the four intervention mechanism types (i.e. channels of engagement), the most commonly employed was convenience $(83 \%, n=33)$, followed by interpersonal $(60 \%, n=24)$, print $(45 \%, n=18)$ and electronic $(25 \%, n=10)$. Interventions were incentivised in $85 \%(n=34)$ of cases, with incentives ranging from practical (e.g. provision of water sample bottle) to financial (e.g. subsidised water treatment), and primarily comprising direct supplementary measures (e.g. provision of water sampling kits) to alleviate well maintenance costs. Pro bono incentives (e.g. free well testing) were reported in $10 \%(n=4)$ of cases. With respect to intervention stakeholders (see Section 2.3), government bodies were the most frequently referenced coordinator type $(48 \%, \mathrm{n}=19)$, followed by educational/research $(45 \%, \mathrm{n}=18)$ and non-profit $(38 \%, \mathrm{n}$ $=15$ ). Community actors (i.e. volunteer groups involved in interventions downstream) were noted in $20 \%(n=8)$ of interventions, all of which were implemented in developing countries.

\subsection{Development status}

A total of 15 identified interventions (38\%) originated from developed (high development) countries and 25 (63\%) from developing (low/medium development) countries (Table 4). More than three quarters of information campaigns $(77 \%, n=20)$ took place in developing countries, while all workshops $(n=4)$ were undertaken in developed countries. A difference approaching statistical significance was found between primary (i.e. overarching) intervention type $\left(\chi^{2}=11.006, \mathrm{p}=0.051\right)$ and development status (Table 6); source installation, source remediation and source treatment-based initiatives were exclusive to developing countries while all interventions entailing workshops as a strategy (primary or otherwise) were documented in developed countries. Over three quarters $(n=19)$ of interventions in developing countries comprised $>1$ intervention type (i.e. multimodal strategies) compared to $27 \%(n=4)$ in developed countries $\left(\chi^{2}=9.337, p=0.002\right)$. Intervention reach was also significantly associated with development category for large-scale interventions $\left(\chi^{2}=6.009, p=0.014\right)$, with $47 \%(n=7)$ of interventions undertaken at this scale in developed countries compared to $12 \%(\mathrm{n}=3)$ in developing countries.

Convenience mechanisms were encountered in all interventions in developing countries $\left(\chi^{2}=14.141, p=<0.001\right)$, with print mechanisms more prevalent in developed countries $(87 \%, \mathrm{n}=13)\left(\chi^{2}=\right.$ 8.095, $p=0.004)$. Presence of an incentive was also significantly associated with development status $\left(\chi^{2}=6.327, p=0.012\right)$; almost all interventions in developing countries $(96 \%, n=24)$ contained $\geq 1$ incentive compared to $67 \%(\mathrm{n}=10)$ in developed countries. In terms of coordinator type, presence of a non-profit body $\left(\chi^{2}=\right.$ $11.111, p=0.001$ ) was significantly associated with development status. Non-profit bodies had a coordinating role in $60 \%(n=15)$ 
Table 3

Characteristics of identified studies $(n=31)$ delineated by country development status.

\begin{tabular}{|c|c|c|c|c|c|c|}
\hline \multirow[t]{2}{*}{ Article } & \multirow[t]{2}{*}{ No. of interventions } & \multirow{2}{*}{$\begin{array}{l}\text { Study location } \\
\text { Country }\end{array}$} & \multicolumn{2}{|c|}{ Contaminants } & \multicolumn{2}{|l|}{ Private groundwater } \\
\hline & & & Type & Origin & Supply type & Supplyregulation \\
\hline \multicolumn{7}{|l|}{ Section 1: Developed $(n=12)$} \\
\hline Benham et al. (2016) & 1 & US & - & - & Drilled, dug, spring & Yes \\
\hline Clemens et al. (2007) & 1 & US & - & - & Drilled & No \\
\hline Flanagan et al. (2015) & 1 & US & Chemical & Geogenic & Drilled, dug & No \\
\hline Flanagan et al. (2016a) & 1 & US & Chemical & Geogenic & - & Yes \\
\hline Flanagan et al. (2016b) & 2 & US & Chemical & Geogenic & - & Yes \\
\hline He et al. (2018) & 1 & US & Chemical & Geogenic & Drilled, dug & Yes \\
\hline Hexemer et al. (2008) & 2 & Canada & Chemical & Geogenic & - & No \\
\hline McCann \& Gold (2012) & 1 & US & - & - & Drilled & Yes \\
\hline Paul et al. (2015) & 1 & US & Chemical & Geogenic & Drilled, dug & Yes \\
\hline Renaud et al. (2010) & 2 & Canada & Chemical & Geogenic & - & No \\
\hline Schubert et al. (1999) & 1 & US & Chemical & Anthropogenic & Drilled & No \\
\hline Swistock et al. (2001) & 1 & US & - & - & Drilled & No \\
\hline \multicolumn{7}{|l|}{ Section 2: Developing $(\mathbf{n}=19)$} \\
\hline Balasubramanya et al. (2014) & 1 & Bangladesh & Chemical & Geogenic & Drilled & No \\
\hline Barnwal et al. (2017) & 1 & India & Chemical & Geogenic & Drilled & No \\
\hline Bennear et al. (2013) & 2 & Bangladesh & Chemical & Geogenic & Drilled & No \\
\hline Brown et al. (2009) & 1 & Cambodia & - & - & Drilled, dug & No \\
\hline Caldwell et al. (2005) & 1 & Bangladesh & Chemical & Geogenic & Drilled, dug & No \\
\hline Chen et al. (2007) & 1 & Bangladesh & Chemical & Geogenic & Drilled & No \\
\hline George et al. (2012) & 2 & Bangladesh & Chemical & Geogenic & Drilled & No \\
\hline George et al. (2013) & 3 & Bangladesh & Chemical & Geogenic & Drilled & No \\
\hline Hadi (2003) & 1 & Bangladesh & Chemical & Geogenic & Drilled & No \\
\hline Hoque et al. (2000) & 1 & Bangladesh & Chemical & Geogenic & Drilled, dug & No \\
\hline Hoque et al. (2004) & 1 & Bangladesh & Chemical & Geogenic & Drilled, dug & No \\
\hline Jakariya et al. (2007) & 1 & Bangladesh & Chemical & Geogenic & Drilled, dug & No \\
\hline Johnston \& Sarker (2007) & 1 & Bangladesh & Chemical & Geogenic & Drilled, dug & No \\
\hline Jones \& Khaira (2014) & 3 & Cambodia & Chemical & Geogenic & Drilled & No \\
\hline Keskin et al. (2017) & 1 & Bangladesh & Chemical & Geogenic & Dug, drilled & No \\
\hline Luby et al. (2008) & 1 & Guatemala & - & - & Dug, spring & No \\
\hline Lule et al. (2005) & 1 & Uganda & Microbial & Anthropogenic & Drilled, dug, spring & No \\
\hline Madajewicz et al. (2007) & 1 & Bangladesh & Chemical & Geogenic & Drilled & No \\
\hline Mahmud et al. (2007) & 1 & Bangladesh & Chemical & Geogenic & Drilled, dug & No \\
\hline
\end{tabular}

Country development status

Developed

Developing

Interventions included

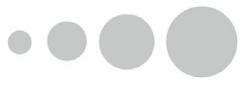

$1 \quad 2-5 \quad 6-9 \quad 10-19$

Figure 3. Global distribution of identified private groundwater interventions (1990-2018). 
Table 4

Intervention characteristics delineated by country development status.

\begin{tabular}{|c|c|c|c|c|c|c|}
\hline Article & $\begin{array}{l}\text { Intervention } \\
*\end{array}$ & $\begin{array}{l}\text { General } \\
\text { attributes } \\
\text { Reach }\end{array}$ & $\begin{array}{l}\text { Constituent } \\
\text { activities } \\
\text { Duration (months) }\end{array}$ & $\begin{array}{l}\text { Stakeholders } \\
\text { Mechanism types \# }\end{array}$ & No. of incentives & Coordinator body type $(s) \neq$ \\
\hline \multicolumn{7}{|l|}{ Section 1: Developed } \\
\hline Benham et al. (2016) & WTS, W & Large & 270 & C, E, I, P & 4 & Edu/Res. \\
\hline Clemens et al. (2007) & $\mathrm{W}$ & Large & 18 & E, I, P & - & Edu/Res., NPO \\
\hline Flanagan et al. (2015) & WTS & Medium & 60 & C, P & 2 & Edu/Res., Govt. \\
\hline Flanagan et al. (2016a) & Reg. & Large & 141 & $\mathrm{P}$ & - & Govt. \\
\hline \multirow[t]{2}{*}{ Flanagan et al. (2016b) } & IC, WTS a & Small & - & $\mathrm{C}$ & 1 & Govt. \\
\hline & IC, WTS $\mathbf{b}$ & Small & - & $\mathrm{C}$ & 1 & Govt. \\
\hline He et al. (2018) & WTS & Large & - & C, E, P & 1 & Edu/Res. \\
\hline \multirow[t]{2}{*}{ Hexemer et al. (2008) } & WTS $^{\mathbf{c}}$ & Medium & 0.23 & C, I, P & 5 & Govt. \\
\hline & WTS $^{\mathbf{d}}$ & Medium & 1 & C, I, P & 5 & Govt. \\
\hline McCann \& Gold (2012) & $\mathrm{W}$ & Large & 196 & E, I, P & 1 & Edu/Res., Govt. \\
\hline Paul et al. (2015) & IC, WTS & Small & 19 & C, I, P & 4 & Other \\
\hline \multirow[t]{2}{*}{ Renaud et al. (2010) } & IC & Small & 3 & $\mathrm{P}$ & 1 & Govt. \\
\hline & IC & Medium & 1 & $\mathrm{E}, \mathrm{P}$ & - & Govt. \\
\hline Schubert et al. (1999) & IC & Large & 48 & $\mathrm{P}$ & - & Govt. \\
\hline Swistock et al. (2001) & $\mathrm{W}$ & Large & 0.03 & E, I, P & - & Edu/Res. \\
\hline \multicolumn{7}{|l|}{ Section 2: Developing } \\
\hline Balasubramanya et al. (2014) & WTS & Medium & 48 & $\mathrm{C}$ & 3 & Govt. \\
\hline Barnwal et al. (2017) & IC, WTS & Medium & 42 & C, I, P & - & Edu/Res. \\
\hline \multirow[t]{2}{*}{ Bennear et al. (2013) } & $\mathrm{IC}^{\mathbf{e}}$ & Medium & 2 & C, I & 1 & Edu/Res. \\
\hline & IC $\mathbf{f}$ & Medium & 2 & C, I & 1 & Edu/Res. \\
\hline Brown et al. (2009) & TSD & Large & 42 & $\mathrm{C}$ & 1 & NPO \\
\hline Caldwell et al. (2005) & IC, WTS & Large & 30 & $\mathrm{C}$ & 1 & Govt., NPO \\
\hline Chen et al. (2007) & IC, SI, WTS & Medium & 46 & C, I & 3 & Edu/Res., Govt., NPO \\
\hline \multirow[t]{2}{*}{ George et al. (2012) } & IC, WTS ${ }^{\mathbf{g}}$ & Medium & 3 & C, I & 3 & Edu/Res., NPO \\
\hline & IC, WTS $\mathbf{h}$ & Medium & 3 & C, I & 3 & Edu/Res., NPO \\
\hline \multirow[t]{3}{*}{ George et al. (2013) } & IC $^{\mathbf{i}}$, WTS & Small & 3 & C, I, P & 2 & Edu/Res., NPO \\
\hline & IC $^{\mathbf{j}}$, WTS & Small & 3 & C, I, P & 2 & Edu/Res., NPO \\
\hline & WTS & Small & 3 & C, P & 2 & Edu/Res., NPO \\
\hline Hadi (2003) & IC, SI, W & Medium & 12 & C, I, P & 1 & Govt., NPO \\
\hline Hoque et al. (2000) & IC, SI, WTS & Small & 12 & C, E, I & 3 & Edu/Res., Govt. \\
\hline Hoque et al. (2004) & IC, SI, W, WTS & Small & 13 & C, I & 4 & NPO \\
\hline Jakariya et al. (2007) & IC, SI, WTS & Medium & 18 & C, E, I & 3 & NPO \\
\hline Johnston \& Sarker (2007) & IC, SI, WTS & Medium & 48 & $\mathrm{C}, \mathrm{P}$ & 3 & Govt. \\
\hline \multirow[t]{3}{*}{ Jones \& Khaira (2014) } & IC, SI, WTS $\mathbf{k}$ & Small & - & C, E, I, P & 3 & NPO \\
\hline & IC, WTS ${ }^{1}$ & Small & - & $\mathrm{C}, \mathrm{P}$ & 2 & NPO \\
\hline & IC, WTS $\mathbf{m}$ & Small & - & C, P & 2 & NPO \\
\hline Keskin et al. (2017) & IC, SI, WTS & Large & 84 & $\mathrm{C}$ & 3 & Govt. \\
\hline Luby et al. (2008) & IC, TSD & Medium & 3 & C, E, I, P & 2 & Govt., Other \\
\hline Lule et al. (2005) & IC, TSD & Medium & 20 & C, I & 2 & Govt. \\
\hline Madajewicz et al. (2007) & IC, SI, WTS & Small & 12 & C, I & 3 & Edu/Res., Govt., NPO \\
\hline Mahmud et al. (2007) & $\mathrm{SR}$ & Medium & 10 & C, I & 3 & Edu/Res. \\
\hline
\end{tabular}

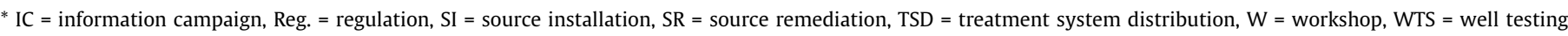
service

\# $\mathrm{C}=$ convenience, $\mathrm{E}=$ electronic, $\mathrm{I}=$ interpersonal, $\mathrm{P}=$ print

‡ Comm. = community, Edu/Res. = educational/research, Govt. = government, NPO = non-profit organisation

Intervention differentiators (as defined by study authors):

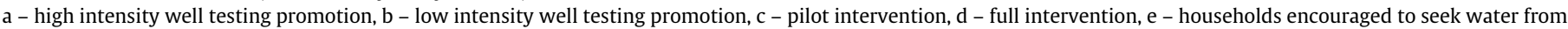

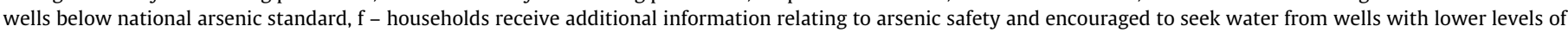

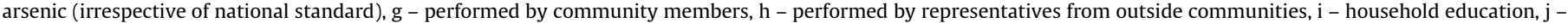
household education and local media campaign, $\mathrm{k}$ - mitigation village, $\mathrm{l}$ - comparison village I, $\mathrm{m}$ - comparison village II

of interventions in developing countries but were noted for only one intervention undertaken in a developed country.

\subsection{Intervention evaluation and efficacy}

Behavioural outcomes were measured in 93\% ( $\mathrm{n}=37)$ and knowledge outcomes in $60 \%(n=24)$ of interventions (Table 5$)$. Outcomes were measured via knowledge, attitudes and/or practices (KAP) surveying in 39 out of 40 intervention evaluations, with the remaining study (Paul et al., 2015) measuring behaviour change via number of well water samples submitted for testing. The most frequent behavioural outcome type was use of a safe water source $(n=14)$, e.g. a newly abstracted well, while risk awareness $(n=17$ ) was the most common type of knowledge outcome.

Discounting outlier values (resulting in truncated mean values), the mean efficacy of all interventions was $45 \%(S D=26.46)$, with interventions in developing countries exhibiting a mean of $44 \%$ $(S D=28.30)$ and developed countries a mean of $47 \%(S D=24.02)$ (Table 7).

\subsection{Behavioural outcomes}

The mean level of behavioural change reported in developed countries was $46 \%(S D=22.49)$ compared to $56 \%(S D=41.70)$ in developing regions. Based on primary intervention type, highest mean behavioural outcomes were recorded for source remediation $(135 \%, \mathrm{n}=1)$, followed by safe source installation $(72 \%, S D=48.85)$ and workshops $(65 \%, S D=16.38)$. In terms of reach, mean behavioural outcomes were highest for small-scale interventions (64\%, $S D=14.18$ ), with large- and medium-reach interventions performing at $55 \%(S D=32.79)$ and $43 \%(S D=35.60)$, respectively.

Mean behavioural outcomes displayed a significant associated with involvement of educational/research coordinators in inter- 
Table 5

Evaluation methods and outcomes (\%) of identified studies delineated by country development status.

\begin{tabular}{|c|c|c|c|c|c|c|c|}
\hline Article & Intervention & Evaluation & Behaviour outcome & Knowledge outcome & & & \\
\hline & Study design * & $\begin{array}{l}\text { Method } \\
\text { Description }\end{array}$ & $\%$ & Description & $\%$ & & \\
\hline \multicolumn{8}{|l|}{ Section 1: Developed } \\
\hline Benham et al. (2016) & WTS, W & Survey & Post & $\geq 1$ action taken $\#$ & +70 & - & - \\
\hline Clemens et al. (2007) & $\mathrm{W}$ & Survey & Post & $\geq 1$ action taken \# & +82 & $\geq 1$ action learned & +92 \\
\hline Flanagan et al. (2015) & WTS & Survey & Post & $\geq 1$ action taken $\#$ & +73 & $\bar{R}$ ecall of well test result & +67 \\
\hline Flanagan et al. (2016a) & Reg. & Survey & Pre-post & Well testing \& treatment & +16 & Recall of well test result & +1 \\
\hline \multirow[t]{2}{*}{ Flanagan et al. (2016b) } & IC, WTS & Survey & Pre-post & Well testing & +32 & Risk awareness & +83 \\
\hline & IC, WTS & Survey & Pre-post & Well testing & +16 & Risk awareness & +52 \\
\hline He et al. (2018) & IC & Survey & Post & Use of safe water source & +29 & - & - \\
\hline \multirow[t]{2}{*}{ Hexemer et al. (2008) } & WTS & Survey & Post & Well testing & +42 & - & - \\
\hline & WTS & Survey & Post & Well testing & +47 & - & - \\
\hline McCann \& Gold (2012) & $\mathrm{W}$ & Survey & Post & Combined actions $\neq$ & +43 & - & - \\
\hline Paul et al. (2015) & IC, WTS & Water samples & Pre-post & Well testing & +243 & - & - \\
\hline \multirow[t]{2}{*}{ Renaud et al. (2010) } & IC & Survey & Pre-post & Well screening & +300 & Risk awareness & -6 \\
\hline & IC & Survey & Post & & - & Risk awareness & +6 \\
\hline Schubert et al. (1999) & IC & Survey & Post & $\geq 1$ action taken \# & +30 & Recall of well test result & +66 \\
\hline Swistock et al. (2001) & W & Survey & Post & $\geq 1$ action taken \# & +66 & $\geq 2$ actions learned & +71 \\
\hline \multicolumn{8}{|l|}{ Section 2: Developing } \\
\hline Balasubramanya et al. (2014) & WTS & Survey & Post & Use of safe water source & +41 & Recall of well test result & +63 \\
\hline Barnwal et al. (2017) & & Survey & Pre-post & Use of safe water source & +31 & Recall of well test result & +50 \\
\hline \multirow[t]{2}{*}{ Bennear et al. (2013) } & IC & Survey & Pre-post & Use of safe water source & +29 & - & - \\
\hline & IC & Survey & Pre-post & Use of safe water source & +20 & - & - \\
\hline Brown et al. (2009) & TSD & Survey & Post & Use of water treatment system & +18 & - & - \\
\hline Caldwell et al. (2005) & IC, WTS & Survey & Pre-post & Combined actions $\ddagger$ & +113 & Risk awareness & +77 \\
\hline Chen et al. (2007) & IC, SI, WTS & Survey & Pre-post & Use of safe water source & +58 & - & - \\
\hline \multirow[t]{2}{*}{ George et al. (2012) } & IC, WTS & Survey & Pre-post & Use of safe water source & +44 & Risk awareness & +68 \\
\hline & IC, WTS & Survey & Pre-post & Use of safe water source & +63 & Risk awareness & +67 \\
\hline \multirow[t]{3}{*}{ George et al. (2013) } & IC, WTS & Survey & Pre-post & Purchase of well test & +93 & Risk awareness & +41 \\
\hline & IC, WTS & Survey & Pre-post & Purchase of well test & +93 & Risk awareness & +41 \\
\hline & WTS & Survey & Pre-post & Purchase of well test & +53 & Risk awareness & +36 \\
\hline Hadi (2003) & IC, SI, W & Survey & Pre-post & & - & Risk awareness & +306 \\
\hline Hoque et al. (2000) & IC, SI, WTS & Survey & Pre-post & Use of safe water source & +144 & - & - \\
\hline Hoque et al. (2004) & IC, SI, W, WTS & Survey & Pre-post & Use of safe water source & +36 & Risk awareness & +78 \\
\hline Jakariya et al. (2007) & IC, SI, WTS & Survey & Post & Use of safe water source & +50 & - & - \\
\hline Johnston \& Sarker (2007) & IC, SI, WTS & Survey & Pre-post & Use of safe water source & +38 & Risk awareness & +71 \\
\hline \multirow[t]{3}{*}{ Jones \& Khaira (2014) } & IC, SI, WTS & Survey & Post & Use of safe water source & +90 & Risk awareness & +42 \\
\hline & IC, WTS & Survey & Post & Use of safe water source & +25 & Risk awareness & +5 \\
\hline & IC, WTS & Survey & Post & & - & Risk awareness & +30 \\
\hline Keskin et al. (2017) & IC, SI, WTS & Survey & Pre-post & Breastfeeding duration & +85 & - & - \\
\hline Luby et al. (2008) & IC, TSD & Survey & Post & Use of treatment system & +3 & - & - \\
\hline Lule et al. (2005) & IC, TSD & Survey & Pre-post & Combined actions $\neq$ & -29 & - & - \\
\hline Madajewicz et al. (2007) & IC, SI, WTS & Survey & Pre-post & Use of safe water source & +60 & Risk awareness & -6 \\
\hline Mahmud et al. (2007) & SR & Survey & Pre-post & Combined actions $\neq$ & +135 & - & - \\
\hline
\end{tabular}

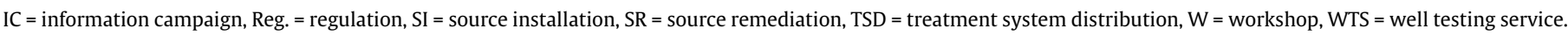

* Pre and post indicates employment of baseline data.

\# Well owners demonstrating adoption of at least one well maintenance behaviour (e.g. periodic testing, supply inspection).

₹ Mean percentage of supply maintenance following amalgamation of percentages for multiple actions.

ventions $(F=5.712, p=0.023)$. Interventions under sole or cosupervision of educational/research coordinators attained a mean behavioural increase of $66 \%(S D=34.29)$ compared to $38 \%(S D=$ 38.67) for those comprising other coordinators. Behavioural outcomes were also significantly associated with involvement of an educational/research coordinator when controlling for study design (i.e. presence of baseline or pre-intervention data) and development status (Table 8). Interventions involving a non-profit organisation in implementation accomplished a mean behavioural outcome of $63 \%$ ( $S D=28.08$ ) while those comprising other coordinator types attained a mean behavioural outcome of $46 \%$ ( $S D=$ 40.044). Interventions involving a government coordinator accomplished a mean behavioural increase of $48 \%(S D=40.68)$ in contrast to $57 \%$ (SD = 32.09) for interventions implemented by different coordinator types (Table 9 ).

Interventions employing $\geq 1$ incentive-based measure (e.g. free well testing) accomplished a mean behavioural change of 54\% (SD $=37.75)$ compared to $45 \%(S D=27.713)$ for interventions foregoing incentives. With respect to intervention mechanisms (Figure 4), highest rates of behaviour were associated with presence of electronic-based mechanisms $(64 \%, S D=40.35)$, followed by interpersonal $(58 \%, S D=39.38)$, convenience $(53 \%, S D=37.93)$ and print $(51 \%, S D=27.59)$ (Fig 5).

\section{Knowledge outcomes}

The mean knowledge outcome reported in developed countries was $48 \%(\mathrm{SD}=37.55)$ compared to $47 \%(\mathrm{SD}=25.75)$ in developing regions. Highest mean knowledge outcomes were attained by interventions comprising workshops $(82 \%, S D=14.85)$, significantly outperforming information campaigns $(41 \%, S D=28.23)$ and well testing services $(47 \%, S D=28.88)$. Large-scale interventions were associated with the greatest increase in knowledge $(61 \%, S D=35.15)$, followed by medium $(56 \%, S D=23.08)$ and small-scale initiatives $(36 \%, S D=29.73)$.

A mean knowledge outcome of $53 \%(S D=26.93)$ was attained by interventions under supervision of an educational/research coordinator compared to $44 \%(S D=32.81)$ for those involving other coordinator types. Interventions under supervision of a governmental organisation in accomplished a mean knowledge outcome 
Table 6

Bivariate analysis of associations between intervention characteristics and development type.

\begin{tabular}{|c|c|c|c|c|}
\hline \multirow[t]{2}{*}{ Variable(s) } & \multicolumn{2}{|c|}{ Development category $^{1}$} & \multirow[t]{2}{*}{ Test statistic $\left(\chi^{2}\right)$} & \multirow[t]{2}{*}{$P$ value } \\
\hline & Developed (\%) & Developing (\%) & & \\
\hline Primary intervention type & & & 11.006 & 0.051 \\
\hline Information campaign & $6(40)$ & $10(40)$ & 0 & 1 \\
\hline Regulation & $1(7)$ & - & 1.709 & 0.191 \\
\hline Source installation & - & $4(16)$ & 2.667 & 0.102 \\
\hline Source remediation & - & $1(4)$ & 0.615 & 0.433 \\
\hline Treatment system distribution & - & $3(12)$ & 1.946 & 0.163 \\
\hline Well testing service & $4(27)$ & $7(28)$ & 0.008 & 0.927 \\
\hline Workshop & $4(27)$ & - & 7.407 & $0.006^{*}$ \\
\hline$>1$ intervention type & $4(27)$ & $19(76)$ & 9.337 & $0.002^{*}$ \\
\hline Intervention reach & & & 6.174 & $0.046^{*}$ \\
\hline Large & $7(47)$ & $3(12)$ & 6.009 & $0.014^{*}$ \\
\hline Medium & $4(27)$ & $13(52)$ & 2.462 & 0.117 \\
\hline Small & $4(27)$ & $9(36)$ & 0.372 & 0.542 \\
\hline Convenience mechanism & $8(53)$ & $25(100)$ & 14.141 & $0.000^{*}$ \\
\hline Electronic mechanism & $6(40)$ & $4(16)$ & 2.88 & 0.09 \\
\hline Interpersonal mechanism & $8(53)$ & $17(68)$ & 1.778 & 0.182 \\
\hline Print mechanism & $13(87)$ & $10(40)$ & 8.335 & $0.004^{*}$ \\
\hline Incentive & $10(67)$ & $24(96)$ & 6.327 & $0.012^{*}$ \\
\hline Edu/res. coordinator & $6(40)$ & $12(48)$ & 0.242 & 0.622 \\
\hline Govt. body coordinator & $10(67)$ & $10(40)$ & 2.667 & 0.102 \\
\hline NPO coordinator & $1(7)$ & $15(60)$ & 11.111 & $0.001^{*}$ \\
\hline
\end{tabular}

${ }^{1}$ Frequency of stated variables by development status.

" Significant at $<0.05$ level.

Table 7

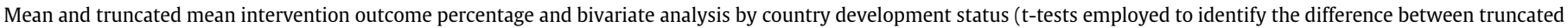
mean outcomes based on country development status).

\begin{tabular}{|c|c|c|c|c|c|c|}
\hline Outcome (\%) & DevelopmentStatus & $\mathbf{N}$ & TruncatedMean $^{1}$ & TruncatedStd. Dev. $^{1}$ & Test statistic $(t)$ & $P$ value \\
\hline \multirow[t]{3}{*}{ Behaviour } & Developed & 14 & 45.50 & 22.49 & & \\
\hline & Developing & 23 & 56.22 & 41.70 & 0.826 & 0.415 \\
\hline & All & 37 & 52.54 & 36.27 & & \\
\hline \multirow[t]{3}{*}{ Knowledge } & Developed & 9 & 48.00 & 37.55 & & \\
\hline & Developing & 15 & 47.36 & 25.75 & -0.049 & 0.961 \\
\hline & All & 24 & 47.61 & 30.80 & & \\
\hline \multirow[t]{3}{*}{ Overall } & Developed & 15 & 47.08 & 24.02 & & \\
\hline & Developing & 25 & 44.18 & 28.30 & -0.309 & 0.760 \\
\hline & All & 40 & 45.26 & 26.46 & & \\
\hline
\end{tabular}

${ }^{1}$ Outliers were identified via box and whisker plots and stem-and-leaf plots in SPSS and removed prior to analysis.

of $43 \%$ (SD $=36.14)$ while those comprising other coordinator types attained a mean behavioural outcome of $52 \%(S D=21.14)$.

No difference in mean knowledge attainment was identified based on presence of absence of incentives, with a mean performance of $48 \%$ recorded interventions with and without incentives $(S D=28.66 ; S D=36.78)$. Interpersonal mechanisms comprised the most successful engagement channel in knowledge attainment ( $54 \%, S D=27.43)$, followed by electronic $(53 \%, S D=37.30)$, convenience $(51 \%, S D=25.11)$ and print based mechanisms. $(41 \%, S D=$ 29.59).

\section{Discussion}

The current study is the first to quantify the efficacy of private well interventions for improving awareness and protective behaviours among private groundwater users. Interventions implemented globally during the period $1990-2018$ were identified via a systematised review and their results pooled with analyses delineated to control for intervention characteristics and country development status (Schardt et al., 2007). Overall, findings indicated that instructor-led, practical interventions allied with both largeand local-scale awareness-raising campaigns may represent an optimum approach for future private well risk prevention initiatives. Further to identifying the current success (and predictors thereof) of pertinent risk mitigation strategies, this study high- lights a series of unique characterisations and limitations in both intervention reporting and scope which also merit reflection.

\subsection{Global distribution of interventions}

The continents of North America, Asia and Africa collectively constitute approximately $80-90 \%$ of the estimated 2.2 billion groundwater users worldwide (Murphy et al., 2017). However, the interventions identified in this review were largely concentrated within the Indian subcontinent and the North-eastern United States, thus accounting for a relatively limited proportion of global private groundwater users. The absence of studies evaluating interventions in Oceania, Europe and South America, may be due to growing urbanisation, lower private groundwater reliance, absence of research-led interventions, publication bias, and warrant future research attention. In some instances, the absence of interventions promoting private well maintenance behaviours and risk awareness may be attributable to government capacity or lack of subsequent "follow through". Bundschuh \& Garcia (2008) and McClintock et al. (2012) note a paucity of data regarding current groundwater arsenic burdens and an absence of remedial interventions in Latin American countries; this in spite of recent estimates placing the number of Latin American populations exposed to chronic concentrations of arsenic at 4.5 million. Significantly, Bundschuh \& Garcia (2008) reference a small number of 
Table 8

Bivariate analysis between intervention outcome efficacy and intervention characteristics.

\begin{tabular}{|c|c|c|c|c|c|c|c|c|}
\hline & $\begin{array}{l}\text { One-way } \\
\text { ANOVA } \\
\text { F-statistic }\end{array}$ & $\begin{array}{l}\text { One-way ANCOVA } \\
\text { (Model 1) } \\
\text { P value }\end{array}$ & $\begin{array}{l}\text { One-way ANCOVA } \\
\text { (Model 2) } \\
\text { F-statistic }\end{array}$ & $\begin{array}{l}\text { One-way ANCOVA } \\
\text { (Model 3) } \\
\text { P value }\end{array}$ & $\begin{array}{l}\text { F- } \\
\text { statistic }\end{array}$ & $\begin{array}{l}\mathbf{P} \\
\text { value }\end{array}$ & $\begin{array}{l}\text { F- } \\
\text { statistic }\end{array}$ & $\begin{array}{l}P \\
\text { value }\end{array}$ \\
\hline \multicolumn{9}{|l|}{ Behaviour } \\
\hline $\begin{array}{l}\text { Primary intervention } \\
\text { type }\end{array}$ & 1.974 & 0.103 & 1.910 & 0.116 & 2.040 & 0.095 & 1.949 & 0.110 \\
\hline$>1$ intervention type & 0.358 & 0.554 & 0.197 & 0.660 & 0.057 & 0.813 & 0.043 & 0.837 \\
\hline Intervention reach & 1.066 & 0.356 & 0.986 & 0.385 & 1.328 & 0.280 & 1.236 & 0.305 \\
\hline $\begin{array}{l}\text { Convenience } \\
\text { mechanism }\end{array}$ & 0.114 & 0.738 & 0.034 & 0.856 & 0.023 & 0.881 & 0.025 & 0.877 \\
\hline Electronic mechanism & 1.241 & 0.273 & 2.400 & 0.131 & 1.933 & 0.174 & 2.693 & 0.111 \\
\hline $\begin{array}{l}\text { Interpersonal } \\
\text { mechanism }\end{array}$ & 1.218 & 0.278 & 1.299 & 0.263 & 0.907 & 0.348 & 1.028 & 0.318 \\
\hline Print mechanism & 0.040 & 0.843 & 0.020 & 0.887 & 0.039 & 0.844 & 0.140 & 0.710 \\
\hline Incentive & 0.247 & 0.623 & 0.210 & 0.650 & 0.035 & 0.852 & 0.055 & 0.816 \\
\hline Edu/res. Coordinator & 5.712 & $0.023^{*}$ & 4.989 & $0.033^{*}$ & 5.575 & $\begin{array}{l}\mathbf{0 . 0 2 4} \\
*\end{array}$ & 5.215 & $\begin{array}{l}\mathbf{0 . 0 2 9} \\
*\end{array}$ \\
\hline $\begin{array}{l}\text { Govt. body } \\
\text { coordinator }\end{array}$ & 0.566 & 0.457 & 0.536 & 0.469 & 0.304 & 0.585 & 0.340 & 0.564 \\
\hline $\begin{array}{l}\text { NPO coordinator } \\
\text { Knowledge }\end{array}$ & 1.882 & 0.179 & 1.673 & 0.205 & 1.192 & 0.283 & 1.261 & 0.270 \\
\hline $\begin{array}{l}\text { Primary intervention } \\
\text { type }\end{array}$ & 1.743 & 0.179 & 1.734 & 0.184 & 1.662 & 0.201 & 1.670 & 0.202 \\
\hline$>1$ intervention type & 0.205 & 0.655 & 0.084 & 0.775 & 0.377 & 0.546 & 0.277 & 0.605 \\
\hline Intervention reach & 1.724 & 0.204 & 1.823 & 0.189 & 1.807 & 0.191 & 1.777 & 0.198 \\
\hline $\begin{array}{l}\text { Convenience } \\
\text { mechanism }\end{array}$ & 0.764 & 0.392 & 0.502 & 0.487 & 1.846 & 0.189 & 1.599 & 0.221 \\
\hline Electronic mechanism & 0.136 & 0.716 & 0.560 & 0.463 & 0.133 & 0.719 & 0.486 & 0.494 \\
\hline $\begin{array}{l}\text { Interpersonal } \\
\text { mechanism }\end{array}$ & 0.898 & 0.354 & 0.658 & 0.427 & 1.013 & 0.326 & 0.825 & 0.375 \\
\hline Print mechanism & 2.294 & 0.145 & 1.695 & 0.208 & 2.336 & 0.142 & 1.706 & 0.207 \\
\hline Incentive & 0.000 & 0.996 & 0.033 & 0.858 & 0.001 & 0.981 & 0.006 & 0.938 \\
\hline Edu/res. Coordinator & 0.495 & 0.489 & 0.323 & 0.576 & 0.497 & 0.489 & 0.343 & 0.565 \\
\hline $\begin{array}{l}\text { Govt. body } \\
\text { coordinator }\end{array}$ & 0.464 & 0.503 & 0.518 & 0.480 & 0.621 & 0.440 & 0.918 & 0.350 \\
\hline NPO coordinator & 0.000 & 0.997 & 0.008 & 0.931 & 0.001 & 0.971 & 0.005 & 0.944 \\
\hline
\end{tabular}

Differences in outcome efficacy by primary intervention type and intervention reach were analysed using One-way ANOVA.

Model 2: One-way ANCOVAs examined differences in outcome controlling for study design across listed variables.

Model 3: One-way ANCOVAs examined differences in outcome controlling for country development status across listed variables.

Model 4: One-way ANCOVAs examined differences in knowledge and outcome controlling for study design and country development status across listed variables.

* Significant at $<0.05$ level.

field studies piloting arsenic removal technologies. Although a number of similar studies were identified in the current literature review (e.g. Cheng et al., 2004; Berg et al., 2006), such interventions were evaluated based on contaminant removal efficiency and human health improvements as opposed to behaviour and knowledge attainment. As noted by Jones-Hughes et al. (2013), interventions premised on installation of contaminant removal technologies may not lead to sufficient, long-term health outcomes in and of themselves and must be better tailored towards (or reported with respect to) behaviour and knowledge acquisition.

\subsection{Reported contaminants}

Notably, the majority of included studies evaluated interventions associated with reducing geogenic (chemical) contaminants in specific "hotspots." The most represented countries in the current review, Bangladesh and the United States, are the subject of historically intensive groundwater arsenic mitigation programmes (Anderson et al., 2015; Henry \& Suk, 2018). Conversely, and of potential concern, prevention of microbial contamination was encountered within just one identified intervention (Lule et al., 2005). This suggests a global lack of appreciation of the scale and severity of groundwater contamination by enteric pathogens and pervasive deficit of remedial interventions (Howard et al., 2006; Bradford \& Harvey, 2017). While the global groundwater arsenic burden is considerable (Ravenscroft et al., 2009), 35-59 million annual cases of enteric infection are attributable to microbial groundwater contamination (Murphy et al., 2017). As information pertaining to the rates and consequent risks of regional microbial groundwater contamination become more widespread, a concurrent increase in correspondent interventions is required to address increasing waste production and climate-related challenges to groundwater quality. Although a number of studies external to this review have indeed evaluated interventions implemented in areas characterised by microbial groundwater contamination (Odagiri et al., 2016), outcomes were premised on improvements in human health and/or contaminant reduction as opposed to behaviour and knowledge attainment.

\subsection{Intervention types}

As is common in awareness raising interventions intended to reach relatively large audiences (Atkin \& Rice, 2012), information campaigns were the most frequently reported intervention type in both development contexts. Interventions in developing countries were typically associated with conventional water and sanitation initiatives often observed in low/medium-development regions such as water testing services and provision of water treatment mechanisms (Mosler, 2012). A greater number of workshopbased initiatives were noted in developed countries in the form of network-based forms of rural outreach delivered by state universities in partnership with federal, state and local governments (Franz, 2014). However, interventions in developed countries were generally less predicated on direct engagement with and actions on behalf of households, with water testing services and provision of treatment mechanisms less frequent. Additionally, interventions 
Table 9

List of variable themes and corresponding types.

\begin{tabular}{l}
\hline Variable Theme \\
\hline Study location \\
Country name \\
Country development status \\
Continent \\
Study area scale \\
Study area population \\
Study area geology \\
Contaminant(s) \\
Total no. \\
Chemical (no/yes) \\
Microbial (no/yes) \\
Anthropogenic source (no/yes) \\
Geogenic source (no/yes) \\
Human health \\
Disease(s)/illness(es) reported (no/yes) \\
No. of disease(s)/illness(es) \\
Type of disease(s)/illness(es) \\
Private groundwater \\
Drilled well (no/yes) \\
Dug well (no/yes) \\
Spring (no/yes) \\
Supply depth \\
Supply figures \\
Regulation (no/yes) \\
Regulation type
\end{tabular}

\section{General intervention attributes}

Intervention type I

intervention type II

intervention type III

Intervention type IV

Reach

Duration in months

Cost stated (no/yes)

Intervention activities

Convenience mechanism (no/yes)

No. of convenience mechanisms

Electronic mechanism (no/yes)

No. of electronic mechanisms

Interpersonal mechanism (no/yes)

No. of interpersonal mechanisms

Print mechanism (no/yes)

No. of print mechanisms

Total no. of mechanisms

Incentive(s) (no/yes)

No. of incentives

\section{Intervention actors}

Community body coordinator (no/yes)

Educational/research body coordinator (no/yes)

Government body coordinator (no/yes)

Non-profit body coordinator (no/yes)

Other type coordinator (no/yes)

No. of coordinators

Community body stakeholder (no/yes)

Educational/research body stakeholder (no/yes)

Government body stakeholder (no/yes)

Non-profit body stakeholder (no/yes)

Other type stakeholder (no/yes)

No. of stakeholders

\section{Intervention evaluation}

No. of evaluation methods

Clinical evaluation (no/yes)

Survey evaluation (no/yes)

Water sample evaluation (no/yes)

Pre-intervention period measured (no/yes)

During intervention period measured (no/yes)

Post-intervention period measured (no/yes)

Pre-intervention period duration in months

During intervention period duration in months

Post-intervention period duration in months

Convenience sample method (no/yes)

Purposive sample method (no/yes)

Table 9 (continued)

\begin{tabular}{ll}
\hline Variable Theme & Variable Type \\
\hline $\begin{array}{l}\text { Random sample method (no/yes) } \\
\text { Representative sample (no/yes) }\end{array}$ & $\begin{array}{l}\text { Dichotomous* } \\
\text { Dichotomous* }\end{array}$ \\
Intervention outcome & \\
Behavioural outcome measure (no/yes) & Dichotomous* \\
Contaminant outcome measure (no/yes) & Dichotomous* \\
Health outcome measures (no/yes) & Dichotomous* \\
Knowledge outcome measure (no/yes) & Dichotomous* \\
Behavioural outcome & Dichotomous* \\
Contaminant outcome & Dichotomous* \\
Health outcome & Dichotomous* \\
Knowledge outcome & Dichotomous* \\
Significant variable & Categorical \\
\hline
\end{tabular}

* Dummy variable.

Dichotomous

Numerical

Categorical

Dichotomous*

Dichotomous*

Dichotomous*

Categorical

Categorical

Dichotomous

Categorical

Categorical

Categorical

Categorical

Categorical

Categorical

Numerical

Dichotomous*

Dichotomous

Numerical

Dichotomous

Numerical

Dichotomous

Numerical

Dichotomous*

Numerical

Numerical

Dichotomous

Numerical

Dichotomous*

Dichotomous*

Dichotomous

Dichotomous

Dichotomous*

Numerical

Dichotomous*

Dichotomous*

Dichotomous*

Dichotomous*

Dichotomous*

Numerical

Numerical

Dichotomous

Dichotomous*

Dichotomous*

Dichotomous

Dichotomous

Dichotomous*

Numerical

Numerical

Numerical

Dichotomous*

Dichotomous* in developed countries adopted convenience mechanisms and incentives significantly more often than developing countries. These findings indicate that private well interventions in developed countries may assume an appreciably greater capacity for private well stewardship and maintenance actions among target audiences. Conversely, opportunities for interpersonal engagement in developing nations may be more frequent due to less secure land title and stigma surrounding private well maintenance (Boyle et al., 2010). It is thus advisable for intervention coordinators in developed countries to engender trust and cooperation with communities from the outset and give greater consideration to the efficacy of convenience, incentive-based approaches (Re, 2015; Morris et al., 2016).

\subsection{Intervention stakeholders}

While the type of documented stakeholders within interventions was not strictly geographically orientated, participation of non-profit bodies was significantly related to development status. Moreover, downstream involvement of community actors (e.g. assistance in information dissemination) was limited to eight interventions, all of which occurred in developing countries. The absence of documented community actors in developed countries may be seen as noteworthy, given that more than half of interventions in developed countries were implemented at the regional- or local scale; interventions undertaken on a sub-national scale may be better placed to utilise community channels to engage private well uses on a more concerted, face-to-face basis (Morris et al., 2016). Furthermore, community and civil society organisations have been repeatedly recognised as an important means of private well user outreach in these regions (Morris et al., 2016; Henry \& Suk, 2018). Significantly, the most frequent coordinator type documented in developed countries was a government body, suggesting that private groundwater interventions in developed countries are generally top-down in nature and may be insensitive to placespecific circumstances. Educational/research bodies were the only consistent coordinator type active in both development categories, suggesting a higher comparable level of expertise and potentially larger funding streams relative to other coordinators with respect to private well user outreach. As educational/research body coordinators were associated with higher behavioural efficacy, they may be best placed to facilitate greater engagement and participation with local, community-based organisations.

\subsection{Intervention outcomes}

Although interventions undertaken in developed countries registered a slightly higher overall success rate than developing coun- 


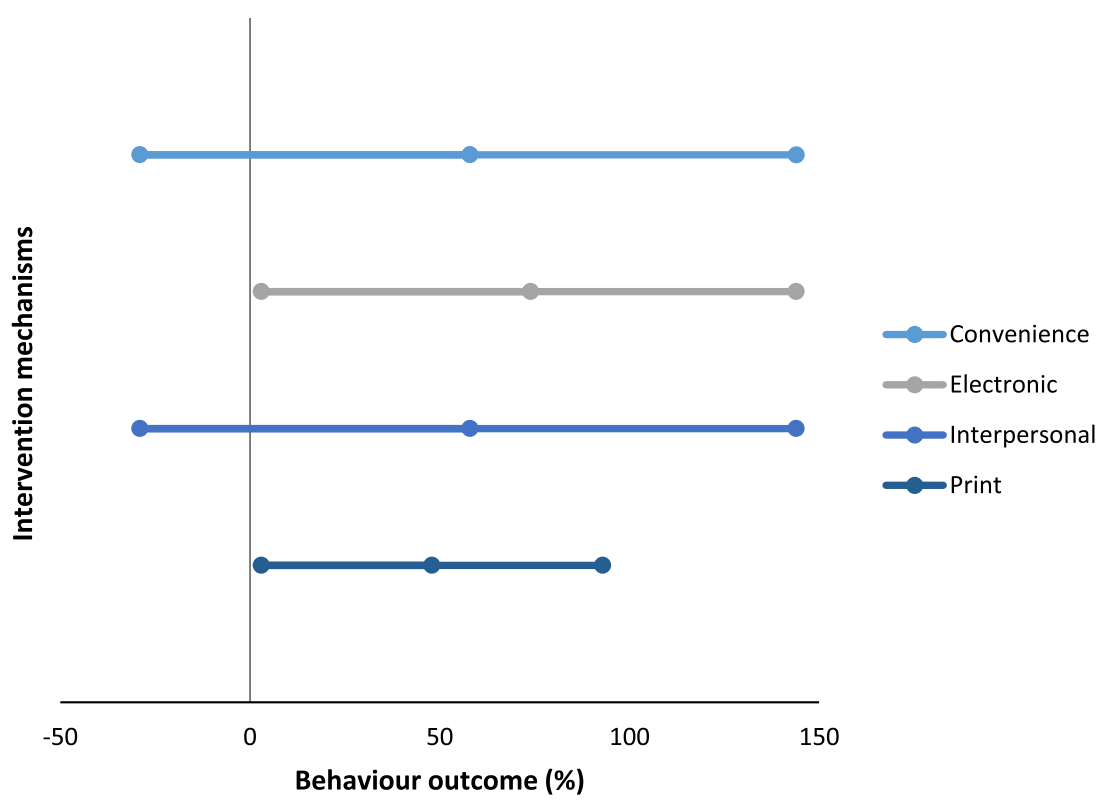

Figure 4. Forest plot demonstrating levels of behavioural attainment (\%) with corresponding intervention mechanisms.

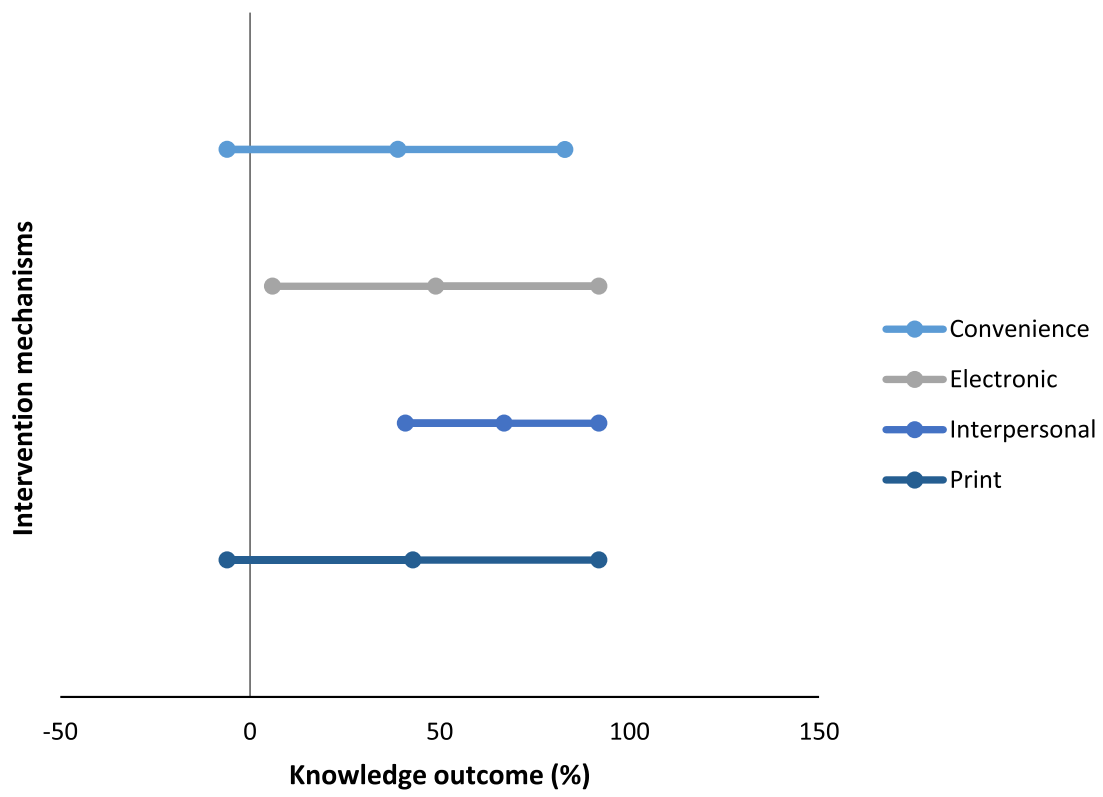

Figure 5. Forest plot demonstrating levels of knowledge attainment (\%) with corresponding intervention mechanisms.

tries ( $47 \%$ vs. $44 \%$ ), mean behavioural outcome was analogous across development type ( $47 \%$ vs. $48 \%$ ). This may be partly ascribed to higher baseline rates of well maintenance (potentially related to higher mean levels of education and income) and/or a lack of assistance relative to interventions in developing countries (Flanagan et al., 2015). This low rate of behaviour change is somewhat similar to previous figures relating to private well user maintenance actions measured in KAP surveys in developed countries, which noted acceptable well maintenance behaviours in approximately $30 \%$ of surveyed well users (Kreutzwiser et al., 2011; Malecki et al., 2017). Highest rates of behaviour change were associated with safe source installation (mean improvement $=135 \%$ ), although workshops (mean improvement $=65 \%$ ) were the most successful primary intervention type in this regard that did not automatically stipulate direct assistance such as well installation or maintenance measures on behalf of householders. The greater success rate in promoting behaviour change compared to information campaigns and well testing services indicates the high productivity of first-hand, interactive interventions in stimulating appropriate private well maintenance. Notwithstanding the validity of these interventions, it is vital that circumstantial and audience-related factors are acknowledged in intervention design as standardised approaches may disregard social, value-based and experiential factors such as frequent correlates with health and water maintenance behaviours (Figueroa \& Kincaid, 2010; Moore \& Boldero, 2017). As participation of educational/research coordinators was also shown to engender significantly higher rates of behaviour change (in both general terms and controlling for development status and study design), their enhanced involvement in subsequent interventions may bear consideration at government level. Involvement of intervention coordinators combining pedagogical and technical expertise may increase the 
likelihood of desired behavioural adaption on the part of well owners, validating calls for greater involvement of educational experts in hydrogeological interventions (Re, 2015; Hynds et al., 2018).

The lower increase in mean knowledge (47\%) attained in developing countries relative to behaviour may be attributable to lower audience literacy rates and a less immediate requirement for information assimilation by virtue of intervention type (e.g. safe source installation undertaken by intervention coordinators/actors) (Jones et al., 2013). Locally adopted interventions attained the highest improvement in behaviour while large-scale interventions attained the highest knowledge increase with respect to audience reach. Highest knowledge outcomes were attained by workshops, further underscoring the efficacy of workshops relative to other intervention types. Two cooperative extension programmes predicated on interpersonal workshops and convenience-based measures (e.g. provision of water sample bottle, well test result interpretation) registered notably high behaviour outcomes (Benham et al., 2016; Clemens et al., 2007). However, as was also observed for behaviour, type of intervention mechanism demonstrated no significant relationship with knowledge outcome. This may denote that efficacy of intervention activities is premised on the quality as opposed to type of engagement material, as noted by Figueroa \& Kincaid (2010) in the context of developing nations. Notably, the majority of knowledge measures were based on awareness of contamination risk as opposed to knowledge of private well maintenance; attainment of $\geq 1$ form of supply maintenance knowledge was measured in only two interventions, once more evincing a lack of focus on well user self-efficacy (Hynds et al., 2018).

The viability of locally scaled interventions in addressing complex human health scenarios (e.g. contaminated domestic water supplies) is repeatedly cited in the health outreach literature and further reinforces the efficacy of locally tailored interventions in promoting enhanced private groundwater quality, stewardship and user health (Craig et al., 2008; Figueroa \& Kincaid, 2010). Conversely, supra-regional (i.e. state/national) interventions such as public communication campaigns may also be required to raise initial awareness or generate discourse; large-scale campaigns performed markedly better than medium-scale interventions. The efficacy of source remediation and safe source installation campaigns and workshops suggests that a combination of household or community-based interventions with interpersonal, practical interventions represent optimal intervention strategies. Such interventions are inherently more effective at the local level but may transcend individual town or village setting if replicated consistently in accordance with an overarching strategy and audience analysis (Milat et al., 2015). On this basis, it is recommended that groundwater outreach practitioners prioritise design and employment of both locally focused, interdisciplinary rural private well interventions and large-scale awareness raising strategies, allied with educational initiatives and tailored awareness-raising campaigns. Concerted and sustained collaboration among stakeholders comprising hydrogeologists, behavioural scientists and community/ voluntary agencies will be necessary to encourage development of feasible, replicable intervention frameworks and, by extension, prevalent rural private groundwater stewardship.

\subsection{Study limitations}

The current review strictly adhered to a systematised review framework and is thus characterised by a number of inherent limitations. As with all scientific reviews, the potential presence of publication bias may impact on the type and location of studies published and reported outcomes. Interventions were largely concentrated within two continental sub-regions, thus potentially limiting the transferability of intervention findings on a global scale. It is further anticipated that a number of interventions may have been undertaken in areas encompassing peri-urban municipalities, in spite of specification of broad rurality. In light of these factors, it is urged that review findings, notwithstanding their potential value, be utilised cautiously.

\section{Conclusion}

Contamination of potable groundwater poses a significant global health threat - one most acutely experienced among rural private owners due to infrequent and inconsistent supply regulation, localised natural and anthropogenic hazards and increased susceptibility to contamination pathways shaped by modern peri-urban/ rural development and climate change dynamics. The pervasive absence of "top-down" supply management via regulation and consequent burden of responsibility placed on well owners themselves prescribes an evidence-based, communicative approach to groundwater risk prevention. The lack of reported interventions in Europe, Australia and South America underscores the potential significance of "place-based" factors such as social geography, policy and institutional capacity/research priorities in determining the occurrence and evaluation of private well interventions. While different geographical regions may require different approaches, the absence of community and voluntary sector coordinators in interventions within developed countries set against requests for their increased involvement therein points to a disconnect that requires attention in intervention design/strategies. This disconnect is also manifest in the distinct lack of private well interventions pertaining to microbial contamination, which warrants increased consideration by governmental and hydrogeological communities alike.

Differences in intervention type (and performance/outcome) with regard to development status may be anticipated given the socio-economic range of target audiences and consequently varying intervention priorities. However, while there is inherent value in examining development categories separately, comparisons may be drawn. Interventions undertaken in developing countries are distinguished by a stronger local focus and a more interpersonal, incentivised-approach. Given the higher overall behaviour rate exhibited among developing country interventions and the observed efficacy of locally-implemented initiatives, an informed adoption of comparable measures in developed countries may result in comparable outcomes. However, higher knowledge rate increases associated with large-scale interventions may indicate the value of a wide geographical reach in generating greater collective discussion and by extension awareness. While the documented efficacy of education/research coordinator-led interventions and workshops reinforces the validity of instructorled interventions, it is nevertheless vital that they are tailored accordingly based on circumstantial factors and audience analysis.

\section{Conflict of Interest}

All authors confirm that no actual or potential conflict of interest exists including any financial, personal or other relationships with other people or organizations within three years of beginning the submitted work that could inappropriately influence, or be perceived to influence, this work.

\section{References}

Anderson, B.E. Naujokas, M.F. Suk, W.A. 2015. Interweaving knowledge resources to address complex environmental health challenges. Environmental Health Perspectives 123, 1095-1099.

Andrade, L., O'Dwyer, J., E., O'Neill, E. \& Hynds, P., 2018. Surface water flooding groundwater contamination, and enteric disease in developed countries: a scoping review of connections and consequences. Environmental pollution 236, 540-549. 
Atkin, C.K., Rice, R.E., 2012. Theory and Principles of Public Communication Campaigns. In: Rice, R.E., Atkin, C.K. (Eds.), Public Communication Campaigns. 4 th ed. Thousand Oaks, CA, SAGE.

Benham, B., Ling, E., Ziegler, P., Krometis, L.A., 2016. Whatâ $€^{\mathrm{TM}} \mathrm{s}$ in Your Water? Development and Evaluation of the Virginia Household Water Quality Program and Virginia Master Well Owner Network. Journal of Human Sciences and Extension 4 (1), 123-138.

Bradford, S.A., Harvey, R.W., 2017. Future research needs involving pathogens in groundwater. Hydrogeology Journal 25 (4), 931-938.

Berg, M., Luzi, S., Trang, P.T.K., Viet, P.H., Giger, W., Stüben, D., 2006. Arsenic Removal from Groundwater by Household Sand Filters: Comparative Field Study, Model, Calculations, and Health Benefits. Environmental Science \& Technology 40, 5567-5573.

Boyle, K.J., Kuminoff, N.V., Zhang, C., Devanney, M., Bell, K.P., 2010. Does a propertyspecific environmental health risk create a "neighborhood" housing price stigma? Arsenic in private well water. Water Resources Research 46, W03507.

Bunn, M.D., Savage, G.T., Holloway, B.B., 2002. Stakeholder analysis for multi-sector innovations. The Journal of Business \& Industrial Marketing 17 (2/3), 181-203.

Charrois, J.W.A., 2010. Private Drinking Water Supplies: Challenges for Public Health. Canadian Medical Association Journal 182 (10), 1061-1064.

Cheng, Z., van Geen, A., Jing, C., Meng, X., Seddique, A., Ahmed, K.M., 2004. Performance OF A Household-Level Arsenic Removal System during 4-Month Deployments in Bangladesh. Environmental Science \& Technology 38, 3442 3448.

Clemens, S.S., Swistock, B.R., Sharpe, W.E., 2007. The master well owner network: volunteers educating Pennsylvania well owners. J. Extension 45 (4).

Craig, P., Dieppe, P., Macintyre, S., Michie, S., Nazareth, I., Petticrew, M., 2008. Developing and evaluating complex interventions: the new Medical Research Council guidance. BMJ 337, a1655.

Elamin, M.B., Flynn, D.N., Bassler, D., Briel, M., Alonso-Coello, P., Karanicolas, P.J., Guyatt, G.H., Malaga, G., Furukawa, T.A., Kunz, R., Schünemann, H., Murad, M.H., Barbui, C., Ciprianij, A., Montori, V.M., 2009. Choice of data extraction tools for systematic reviews depends on resources and review complexity. Journal of Clinical Epidemiology 62, 506-510.

Falagas, M.E., Pitsouni, E.I., Malietzis, G.A., Pappas, G., 2008. Comparison of PubMed, Scopus, Web of Science, and Google Scholar: strengths and weaknesses. The FASEB Journal 22 (2), 338-342.

Figueroa, M.E., Kincaid, D.L., 2010. Social, Cultural and Behavioral Correlates of Household Water Treatment and Storage. Center Publication HCI 2010-1: Health Communication Insights. Johns Hopkins Bloomberg School of Public Health. Center for Communication Programs, Baltimore, MD.

Franz, N.K., 2014. Measuring and Articulating the Value of Community Engagement: Lessons Learned from 100 Years of Cooperative Extension Work. Journal of Higher Education Outreach and Engagement 18 (2), 5-16.

Flanagan, S.V., Marvinney, R.G., Johnston, R.A., Yang, Q., Zheng, Y., 2015. Dissemination of well water arsenic results to homeowners in Central Maine: influences on mitigation behavior and continued risks for exposure. Science of the Total Environment 505, 1282-1290.

Gleeson, T., Befus, K.M., Jasechko, S., Luijendijk, E., Bayani Cardenas, M., 2016. The global volume and distribution of modern groundwater. Nature Geoscience 9, 161-167.

Gorelick, S.M., Zheng, C., 2015. Global change and the groundwater management challenge. Water Resources Research 51, 3031-3051.

Green, T.R., Taniguchi, M., Kooi, H., Gurdak, J.J., Allen, D.M., 2011. Beneath the surface of global change: Impacts of climate change on groundwater. Journal of Hydrology 405, 532-560.

Haddaway, N.R., Collins, A.M., Coughlin, D., Kirk, S., 2015. The Role of Google Scholar in Evidence Reviews and Its Applicability to Grey Literature Searching. PLoS ONE 10, (9) e0138237.

Henry, H.F., Suk, W.A., 2018. Public Health and Karst Groundwater Contamination: From Multidisciplinary Research to Exposure Prevention. In: White, W.B. Herman, J.S., Herman, E.K., Rutigliano, M. (Eds.), Karst Groundwater Contamination and Public Health. Basel, Springer Nature, pp. 7-14.

Higgins, J.P.T., Green, S. (Eds.), 2011. Cochrane Handbook for Systematic Reviews of Interventions, Version 5.1.0. Cochrane, London.

Howard, G., Bartram, J., Pedley, S., Schmoll, O., Chorus, I., Berger, P., 2006. Groundwater and public health. In: Schmoll, O., Howard, G., Chilton, J. Chorus, I. (Eds.), Protecting Groundwater for Health: Managing the Quality of Drinking-water Sources. IWA, London.

Hynds, P.D., Thomas, M.K., Pintar, K.D., 2014. Contamination of groundwater systems in the US and Canada by enteric pathogens, 1990â€"2013: a review and pooled-analysis. PLoS One. 9, (5) e93301.

Hynds, P., Regan, S., Andrade, L., Mooney, S., O’Malley, K., DiPelino, S., O’Dwyer, J., 2018. Muddy Waters: Refining the Way forward for the "Sustainability Science" of Socio-Hydrogeology. Water 10 (9), 1111.

Jakeman, A.J., Barreteau, O., Hunt, R.J., Rinaudo, J.D., Ross, A., Arshad, M., Hamilton, S., 2016. Integrated Groundwater Management: An Overview of Concepts and Challenges. In: Jakeman, A.J., Barreteau, O., Hunt, R.J., Rinaudo, J.D., Ross, A. (Eds.), Integrated Groundwater Management: Concepts, Approaches and Challenges. Springer, New York City, NY.

Jones, S.A., Sanford Bernhardt, K.L., Kennedy, M., Lantz, K., Holden, T., 2013. Collecting Critical Data to Assess the Sustainability of Rural Infrastructure in Low-Income Countries. Sustainability 5 (11), 4870-4888.

Kreutzwiser, R., de Loë, R., Imgrund, K., Conboy, M.J., Simpson, H., Plummer, R., 2011. Understanding stewardship behaviour: Factors facilitating and constraining private water well stewardship. Journal of Environmental Management 92, 1104-1114.

Lapworth, D.J., Baran, N., Stuart, M.E., Ward, R.S., 2012. Emerging organic contaminants in groundwater: A review of sources, fate and occurrence. Environmental Pollution 163, 287-303.

Lule, J.R., Mermin, J., Ekwaru, J.P., Malamba, S., Downing, R., Ransom, R., et al., 2005. Effect of home-based water chlorination and safe storage on diarrhea among persons with human immunodeficiency virus in Uganda. American Journal of Tropical Medicine and Hygiene 73, 926-933.

Malecki, K.M.C., Schultz, A.A., Severtson, D.J., Anderson, H.A., VanDerslice, J.A., 2017. Private-well stewardship among a general population based sample of private well-owners. Science of the Total Environment 601-602, 1533-1543.

Milat, A.J., Bauman, A., Redman, S., 2015. Narrative review of models and success factors for scaling up public health interventions. Implementation Science 10,113.

Mitchell, M., Curtis, A., Sharp, E., Mendham, E., 2012. Directions for social research to underpin improved groundwater management. Journal of Hydrology 448449, 223-231.

Moher, D., Liberati, A., Tetzlaff, J., Altman, D.G., 2009. Preferred Reporting Items for Systematic Reviews and Meta-Analyses: The PRISMA Statement. PLoS Medicine 6, (7) e100009.

Moore, H.E. \& Boldero, J. (2017) Designing Interventions that Last: A Classification of Environmental Behaviors in Relation to the Activities, Costs, and Effort Involved for Adoption and Maintenance. Frontiers in Psychology, 8: 1874.

Morris, L., Wilson, S., Kelly, W., 2016. Methods of conducting effective outreach to private well owners - a literature review and model approach. Journal of Water and Health 14 (2), 167-182.

Mosler, H.J., 2012. A systematic approach to behavior change interventions for the water and sanitation sector in developing countries: a conceptual model, a review, and a guideline. International Journal of Environmental Health Research 22 (5), 431-449.

Murphy, H.M., Prioleau, M.D., Borchardt, M.A., Hynds, P.D., 2017. Review: Epidemiological evidence of groundwater contribution to global enteric disease, 1948-2015. Hydrogeology Journal 25 (4), 981-1001.

Niu, B., Loáiciga, H.A., Wang, Z., Benjamin Zhan, F., Hong, S., 2014. Twenty years of global groundwater research: A Science Citation Index Expanded-based bibliometric survey (1993-2012). Journal of Hydrology 519 (A), 966-975.

Odagiri, M., Schriewer, A., Daniels, M.E., Wuertz, S., Smith, W.A., Clasen, T. Panigrahi, P., 2016. Human fecal and pathogen exposure pathways in rural Indian villages and the effect of increased latrine coverage. Water research 100 232-244.

O’Dwyer, J., Morris Downes, M., Adley, C.C., 2016. The impact of meteorology on the occurrence of waterborne outbreaks of vero cytotoxin-producing Escherichia coli (VTEC): a logistic regression approach. Journal of Water and Health 14 (1), 39-46.

Oxman, A.D., Lavis, J.N., Lewin, S. \& Fretheim, A. (2009) SUPPORT Tools for evidenceinformed health Policymaking (STP) 10: Taking equity into consideration when assessing the findings of a systematic review. Health Research Policy and Systems, 7(1): S10.

Paul, M.P., Rigrod, P., Wingate, S., Borsuk, M.E., 2015. A community-driven intervention in Tuftonboro, New Hampshire, succeeds in altering water testing behavior. Journal of environmental health 78 (5), 30.

Pearson, M., Jones-Hughes, T., Whear, R., Cooper, C., Peters, J., Evans, E.H., Depledge, M., 2011. Are interventions to reduce the impact of arsenic contamination of groundwater on human health in developing countries effective?: a systematic review protocol. Environmental Evidence 1, 1.

Ravenscroft, P., Brammer, H., Richards, K., 2009. Arsenic Pollution: A Global Synthesis. John-Wiley \& Sons, Oxford.

Re, V., 2015. Incorporating the social dimension into hydrogeochemical investigations for rural development: the Bir Al-Nas approach for sociohydrogeology. Hydrogeology Journal 23 (7), 1293-1304.

Rowe, G., Frewer, L.J., 2005. A Typology of Public Engagement Mechanisms. Science Technology, \& Human Values 30 (2), 251-290.

Schardt, C., Adam, S., M.B., Owens, T., Keitz, S. \& Fontelo, P. (2007). Utilization of the PICO framework to improve searching PubMed for clinical questions. BMC Medical Informatics and Decision Making, 7: 16.

Schwarzenbach, R.P., Egli, T., Hofstetter, T.B., von Gunten, U., Wehrli, B., 2010. Global Water Pollution and Human Health. Annual Review of Environment and Resources 35, 109-136.

Shankar, S., Shanker, U., Shikha, 2014. Arsenic Contamination of Groundwater: A review of sources, prevalence, health risks, and strategies for mitigation. The Scientific World Journal 2014, 304524.

UNDP, 2016. Human Development Report 2016: Human Development for Everyone. United Nations Development Programme, New York City, NY.

Valle Junior, R.F., Varandas, S.G.P., Sanches Fernandes, L.F., Pachecho, F.A.L., 2014 Groundwater quality in rural watersheds with environmental land use conflicts. Science of the Total Environment 493, 812-827.

Waddington, H., White, H., Snilstveit, B., Garcia Hombrados, J., Vojtkova, M., Davies, P., Bhavsara, A., Eyers, J., Perez Koehlmoos, T., Petticrew, M., Valentine, J.C. Tugwell, P., 2012. How to do a good systematic review of effects in international development: a tool kit. Journal of Development Effectiveness 4 (3), 359-387.

WHO, 2009. Practical Guidance for Scaling up Health Service Innovations. World Health Organization, Geneva.

WHO/UNICEF (2015) 25 Years Progress on Sanitation and Drinking Water: 2015 Update and MDG Assessment. New York, NY: United Nations Children's Fund and World Health Organisation. 